\title{
Ein Beitrag zur Chirurgie des Gehirns.
}

Von Dr. J. Ruhemann in Berlin.

In No. 16 , S. 251 , dieser Wochenschrift berichtet $\mathrm{Hahn}$ über das Schicksal eines Mannes, den er zweimal operirte und den ich 14 Tage vor dem ersten chirurgischen Eingriff auf gütige Veranlassung des Augenarztes Dr. G. Gutmann untersuchte, und zwar am 28. December 1891. Ich theile zunächst ganz kurz den von mir erhobenen Befund mit.

Der 30 jährige Schlächter A. P. klagt seit circa zwei Jahren über häufig auftretenden occipito-frontalen Kopfschmerz, Schwindelanfälle, Brechneigung und Brausen in den Ohren. Seit October bemerkt er schnelle Abnahme der Sehkraft. Rechts Staungsspapille, links neuritische Atrophie, besonders der temporalen Hälfte. Gedächtnissschwäche, keine Sprachstörung.

Prüfung der Motilität ergiebt Schwanken beim Stehen und Gehen, falls die Augen geschlossen werden. Die sehr erweiterten Pupillen reagiren schwach auf Licht. Die Stirn zeigt Percussionsschmerzhaftigkeit. Patellarreflexe vorhanden. Sensibilität intact.

Ich nahm auf Grund des Kopfschmerzes, des Schwindels, des schwankenden Ganges, des Erbrechens und des Augenbefundes das Vorbandensein eines Kleinhirntumors (Cysticercus oder Echinococcus) an.

Da der Kopfschmerz immer intensiver wurde, so wurde der Kranke Prof. Hahn überwiesen, der dann am 11. Januar 1892 die temparäre Resection an der linken Stirnhälfte vornahm, ,weil im linken Hirnlappen (Stirnlappen?) ein raumbeengender Process, und zwar Cysticercus angenommen wurde". 1) Die Punction ergab $120 \mathrm{ccm}$ einer klaren Flüssigkeit. Hiernach verloren sich die Beschwerden. Es trat Besserung der Sehkraft des rechten Auges ein. Nach der Operation wurde von $\mathrm{Hahn}$ die Wahrscheinlichkeitsdiagnose auf Tumor mit Druck auf die Vena magna Galeni gestellt, also entsprechend meiner anfangs gestellten Diagnose auf Kleinhirngeschwulst, durch die ja häufig auf jene Weise Hydrocephalus internus bedingt wird. Nach $1^{1 /} / 2$ Jahren andauernder Besserung stellten sich be dem Patienten die unerträglichen Kopfschmerzen wieder ein; jetzt waren ataktische Beschwerden unverkennbar. Lumbalpunction. Zwei Tage später Exitus.

Die Section ergab einen derbwandigen cystischen Tumor von Haselnussgrösse im vorderen Theil der stark erweiterten Rautengrube und sehr starken Hydrocephalus internus.

Ich bemerke, dass die von mir vor der ersten Operation gestellte Diagnose der Wirklichkeit am nächsten kam. Aber die Kleinhirnsymptome (Cerebellarataxie, Hydrocephalus) waren das Resultat einer Fernwirkung, wobei es erstaunlich erscheint, dass ein verhältnissmässig so kleiner Tumor an solcher Stelle seine Druckwirkung nur nach oben, nach dem Kleinhirn zu entwickelte. Die harte Geschwulst wurde nämlich, nachdem der durch Ependymreizung hervorgerufene Hydrocephalus internus eine gewisse Grösse erreicht hatte, auf dem schwer comprimirbaren Ventrikelwasserkissen schwimmend wie ein Pelottenknopf gegen die Vena magna Galeni angepresst, wodurch wiederum die Anstauung des Liquor cerebrospinalis unterhalten wurde. Wäre meine Ansicht maassgebend gewesen, so würde man, falls nicht von jedem Eingriff abgerathen worden

1) Vergl. Centralblatt für Augenheilkunde 1893, S. 143 u. 169. 
wäre, das Kleinhirn als Operationsfeld in Anspruch genonmen liaben. Das Resultat wäre negativ gewesen, während die gegen das Hauptsynıptom gerichtete chirurgische Procedur von gutem Erfolge begleitet war, obwohl sie olıne Zugrundelegung der eigentlichen Diagnose vorgenommen wurde. Darf man eine Consequenz ans diesem Falle ziehen, so ist es diese, dass man in Fällen von Kleinhirngeschwälsten oder von das Cerebellum comprimirenden Tumoren, wo hydroceplialische Erscheinungen anzunehmen sind, zunächst dio Gehirnpunction vornimmt und den Lrfolg abwartet, bevor man an die so wenig chancenreiche Entfermung der Kleinhirntumoren herangeht, seien sie noch so sicher diagnosticirt und erweisen sie sich selbst als wirklich operabel. 